\title{
How Micro Current Created by Grounding Stimulates Meridian Points in Acupressure?
}

\author{
Madiha Khalid and Jeremy Madvin
}

\section{ABSTRACT}

\begin{abstract}
Acupressure in Oriental medicine is used as a core therapeutic method due to its therapeutic efficacy and minimal side-effects. However, microcurrent stimulation, combined with acupressure and grounding, has not been studied under acupressure conditions in terms of how this combination might affect acupressure therapy's effectiveness. This paper clarifies the new concept of acupressure stimulation combined with a microcurrent that generates electron flow during earthing. In grounding the body, there is free electron movement from and to the body that creates microcurrent. Acupressure uses these currents to activate specific acupoints for therapeutic purposes and activating these acupoints adjust the imbalance between Qi across channels (meridians) and ultimately treat diseases. It is time for proper research and implementation of this technique to increase acupressure efficacy and reduce society's cost.
\end{abstract}

Keywords: Acupoints, Acupressure, Earthing, grounding, meridian points, microcurrent.
Submitted : March 03, 2021

Published : March 31, 2021

ISSN: $2593-8339$

DOI: $10.24018 /$ ejmed.2021.3.2.749

\section{Dr. Madiha Khalid *}

Humdard University, Islamabad Campus, Pakistan.

(e-mail: msmadihakhalid@gmail.com) Jeremy Madvin

MBA International Business University of Redlands, United States.

(e-mail: jeremymadvin ${ }^{\circledR}$ gmail.com)

*Corresponding Author

\section{INTRODUCTION}

Acupressure is a modified form of acupuncture that substitutes needle insertion with pressure. Acupressure has originated from ancient China [1]. Its principle works from activation of acupoints through the meridians with fingers or non-invasive tools that exert pressure on the point. Because of improved awareness of the benefits of various holistic approaches, this hands-on therapy is used more frequently than before [2].

Meridians are the channels inside the human body that help retain Qi and interconnect to the human body's various tissues and organs. Its activation induces an analgesic effect both locally and systematically when applying pressure on a particular point [3]. Acupoint is the point nearest to the skin surface, and the human body is full of clusters of such elements [4]. There are particular points with high electrical conductance used in acupuncture for needle insertion on the body's surface [5]. Acupoints are electrically active only when there is a dysfunction in the body. It appears that the size and shapes of acupuncture points differ considerably. The acupoint position on a defined meridian is measured using a body inch (BI) or Cun. One BI/Cun is equal to one thumb width at the base of the fingernail. The acupressure measurement units (AUM) are known as BI/Cun [6]. Various acupoints in the zone appear to become active in different situations. The points are dynamic, becoming particularly active after injury or disease.

Qi base on the philosophy of the energy life force, but consistency defines one's health status. According to Traditional Chines Medicine (TCM), acupuncture uses energy to activate specific acupoints for therapeutic purposes and activate these acupoints adjust the imbalance between Qi across channels (meridians) and ultimately treat diseases [7].

Acupressure may be paired with grounding techniques to maximize the possible health benefits; grounding or earthing applies to the skin's direct contact with the Earth's surface. The grounding effect is achieved by using bare feet or hands or using various grounding devices.

Recent research has shown that when the human body electrically contacts Earth's surface, it affects different processes such as inflammation, wound healing, and immune responses; this technique can control chronic inflammatory and autoimmune diseases [8].

Grounding systems are simple conductive systems that can easily be used within the home or office in the form of mats, sheets, wrist or ankle bands, sticky patches, and other various comfortable and convenient, user-friendly methods. Such systems connect to the Ground via a wire [8].

Microcurrent therapy (MT) means clinical uses of very low electric current $(<1 \mathrm{~mA})$, which is typically sub-sensory for the body [9]. Microcurrent therapy supporters believe that the body can more effectively accept electrical energy into its electrophysiological system by reducing the current to a subsensory level to boost and promote tissue healing and regeneration [10], [11]. It speculates that microcurrent stimulation significantly improves the bodies healing process by increasing cellular physiology and growth and biochemical osmosis [11]. In this paper, I want to clarify the new concept of stimulation of acupressure points with microcurrents that generate with the flow of electro during grounding. 


\section{MODERN CONCEPTS OF MiCROCURRENT STIMULATION WITH EARTHING AND MERIDIANS}

Modern science has confirmed that every living thing has electrical charges inside its body. The human body is no exception. Modern physiology tells us that these charges', the strength of charges, and the distribution of charges can be measured despite their small sizes.

The electrical energy of the human body was first measured systematically by Dr. Reinhold Voll from Germany. Voll measured electrical resistance at various points of the body with the help of an electrical probe. He revealed several points in the body that gave unusual readings, lower electric resistance, and those points distributed over fixed routes [12].

At the same time, Nakatani, a Japanese doctor, used an electrical device for examining patients. Nakatani also identified several low electrical resistance points, which he associated in routes(13). These electrical pathways are almost identical to the meridians of traditional Chinese medicine. Furthermore, the points of low electrical resistance exactly match with acupuncture points. Electrical current flow through those low resistance routes is called Chi.

The higher organism typically includes the digestive, circulatory, nervous, and reproductive systems. There may be another system that correlates to the low electrical resistance pathways - meridians located under the skin and connected to the internal organs [13].

Acupoints are similar to boost stations in the meridians that link acupuncture points with these meridian lines to transmit these DC signals. Acupoints in meridians have different electrical properties, and these properties are used for diagnostic purposes.

Acupoints show little or no electrical activity when the tissue or organ is healthy [14]. If injury or disease occurs, a current is created locally at the damage site [15]. Simultaneously, in relationship with the surrounding skin, the corresponding acupoint changes the characteristics and the polarity. Acupuncture points related to the injury site are typical, although not always usually located far away.

For this understanding of the mechanism of acupuncture, the idea of semi-conduction is fundamental. One of the pioneers of relevant modern concepts, Albert Szent Gorgyi, proposes ionic conduction to conduct energy using charged particles (ions) [17]. Nerve impulses are carried out in this way. Ionic currents are well functioning over short distances, such as a membrane that covers nerve fibers but quickly dissipates over longer distances. Semi-conduction produces and induces a current and involves a highly orderly structure of materials so that electrons can pass from one nucleus to another. Semi-conductors work both as insulators and conductors, depends on temperature. They can carry only small currents, but the current can transport efficiently over long distances.

This concept can comprise various conventional electric circuits, with the most descriptive being the diode gate. A diode gate is considered a building block of a microprocessor. In the case of injury, the semi-conductor effect restricts the free flow of charge leading to accumulation of charges and hence causes pain [16].

The concept of a diode that is a semi-conductor that only allows current to go in a single direction is central to the fundamental explanation of acupuncture's working [15]. The following diagrams (Fig. 1) illustrates this:

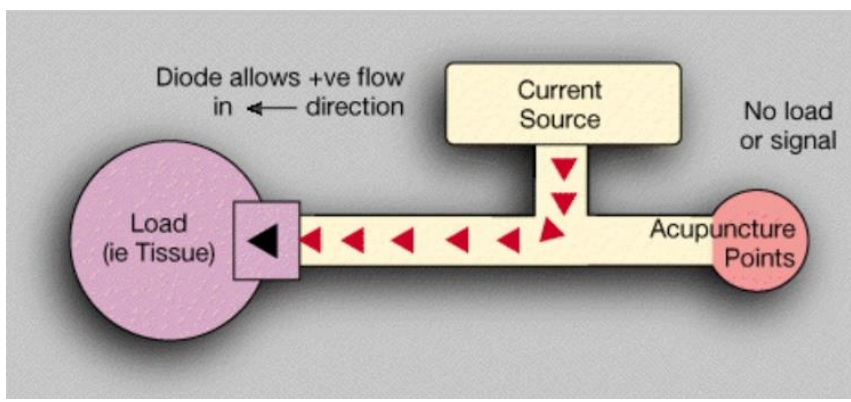

Fig. 1. When the tissues are healthy.

The current source is the metabolism of the body itself. The load is the body part that is attached to a particular acupoint shown on the diagram. The acupuncture point does not show any change if the load or the tissue is healthy.

In Fig 2, when the load is injured, a current of injury generates. The current flows into that area are blocked. It results in the current flow back from the current source, and it activates the acupoint.

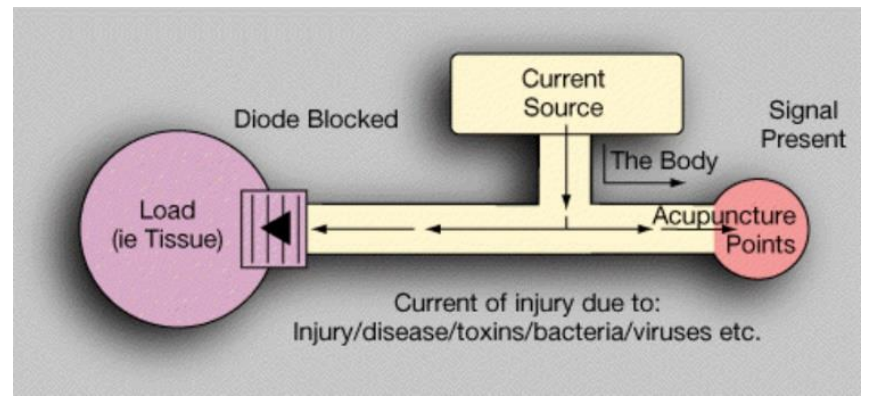

Fig. 2. In case of injury.

Fig. 3 shows the use of biphasic signals (grounding) for the treatment of acupuncture points.

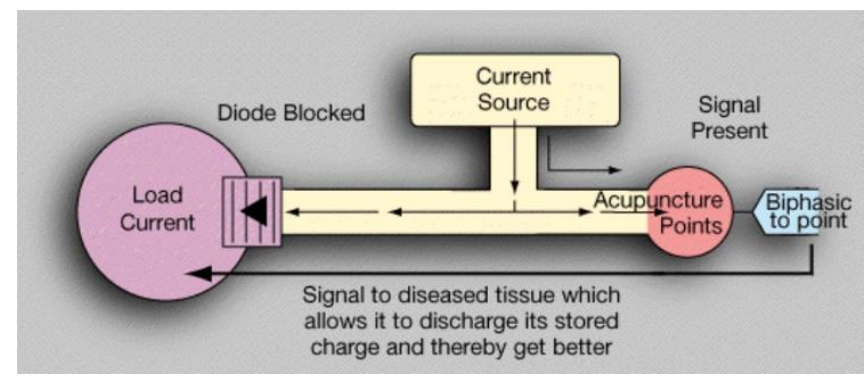

Fig. 3. When grounding takes place.

Acupuncture points are generally negatively charged from the surrounding skin. Higher negative values represent increased electrical activity in the corresponding anatomical area. It is found in functional pathology, where there is no actual organic change or damage. This level can increase in acute conditions, and as this level increases, the is a correlation associated with severe pain [15].

One will find that if a bipolar electric current passes through a semi-conducting tissue, typical conductive properties are recovered, the stored charges will release from the damaged area, and the symptoms of the disease reduce. Sometimes with sufficient therapy, the problem can disappear completely. 
Current research indicates that the human body's grounding has a significant impact on bioelectrical and bioenergetic mechanisms and seems to have a significant modulating effect on chronic diseases and dysfunction [19].

Furthermore, Oschman and colleagues proposed that a connection between the body and the Earth causes free electrons from the Earth's surface to transfer to the body, where they can have antioxidant effects [8]. Additional studies to support these theories are needed.

\section{WHY MICROCURRENT?}

In grounding the body, there is free electron movement from and to the body that creates a microcurrent in the body. This microcurrent helps heal different processes that are best explained by the gate control theory. The Melzack and wall [20] gate control theory is the best justification for the use of high-intensity milliampere stimulation. The high-intensity current stimulation tends to block gates to the associated nerve impulses, according to Melzack and wall [20]. Specifically, stimulation of the beta-sensory neurons, which respond faster than the smaller pain-transmitting C-fibers, would tend to stop the conduction of pain signals to the spinal cord in the substantia gelatinosa. The beta sensory input effectively floods the circuits and blocks the C-fibers' pain signals from getting through. The secretion may also enhance this presynaptic feedback inhibition in endorphins and enkephalins' central nervous system, the body's naturally occurring narcotics. High-intensity electrical stimulation would irritate the tissues enough to activate the protective response of these endogenous opiates. Such relief from pain will last for many hours. Unfortunately, such effects represent only temporary masking of the pain without any long-lasting cumulative effects and without any direct impact on the body's healing process [20]. However, with microcurrent stimulation, it is proposed that instead of masking the pain of injury, there may be a way to initiate the body's natural healing process via the electrophysiological system [20]. The benefits of microcurrent claim to be the cumulative treatment effects on pain. Those using microcurrent are familiar with the next-day carry-over. Patients may not notice any immediate analgesia, but the next day reports remarkable subjective improvement corroborated by objective examination revealing reduced pain with palpation, diminished swelling, normalization of skin colouration, and improved motion range [11].

\section{ACUPRESSURE AND GROUNDING DEVICES}

There is a wide variety of portable acupressure and grounding devices available in the market. Most acupressure devices exert constant pressure on specific acupoints. Due to a nerve accommodation limitation, automated modulating acupressure devices have been introduced in the market to increase the therapeutic effect.

In one study, Mehta et al. [6], the authors summarized the principal forms of acupressure devices (Fig. 4), and they can pair with grounding to achieve synergistic benefits.

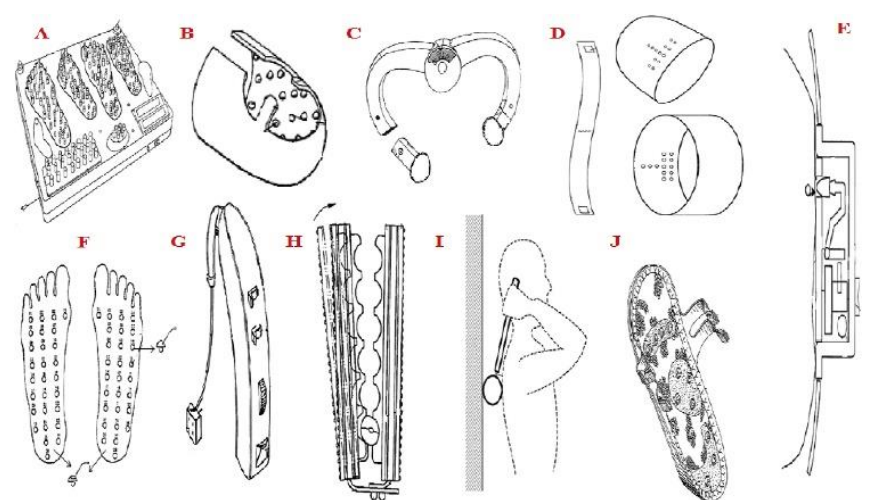

Fig. 4. (Metha et al.). Patented devices (A-J). Acupressure device name: A. Acupressure footboard, B. Acupressure device for fingers, C. Acupressure and reflexology clamp, D. Acupressure device for treating insomnia, E. Automatically modulating acupressure device, F. Adjustable foot acupressure and pain relief platform, G. Electrical plus acupressure system, H. Finger acupressure apparatus, I. Hand-held acupressure device, and J. Massage footwear.

However, very few devices provide combined effects of acupressure with grounding. An instrument that can fulfil this need can be a modified acupressure probe with a conductive grounding cable on the one hand and a tip for applying a specific level of pressure on specific points along meridians on the other hand (Fig. 5).

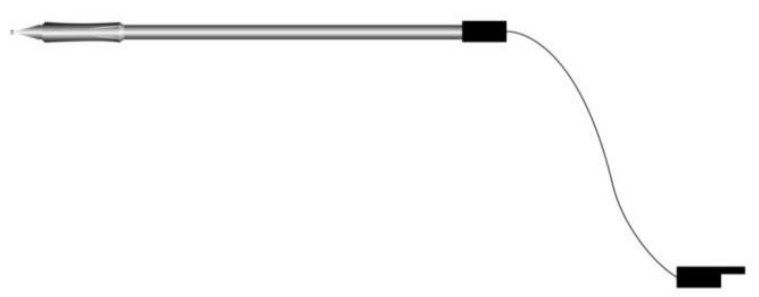

Fig. 5. Schematic device for combining acupressure and grounding techniques.

\section{Discussion}

Both grounding acupressure and acupressure are simple, safe, effective, and cost-effective treatments, but due to the grounding acupressure's dual nature, it seems more efficient and effective than either earthing or grounding alone. These two complementary therapies provide many health benefits that will provide a basis for more research.

The first effects analyzed as a result of therapies are inflammation and immunological responses. Several studies have shown advantages for both grounding and acupressure over the past several years, so the synergistic effects could be more efficient to reduce this form of immunological response.

In a study by Oschman et al., the authors have shown that grounding can relieve pain, change the number of circulating immune cells (neutrophils and lymphocytes), and change the circulating chemical factors associated with inflammation (Fig. 6) [8]. This result shows that the Earth has significant anti-inflammatory effects. An explanation of the theory may be the reason that grounding enables a large number of free electrons to enter the body through nervous, meridian, and circulatory systems so that free radicals, created by our metabolism, that have damaging effects on cells can be neutralized [21]-[22]. 

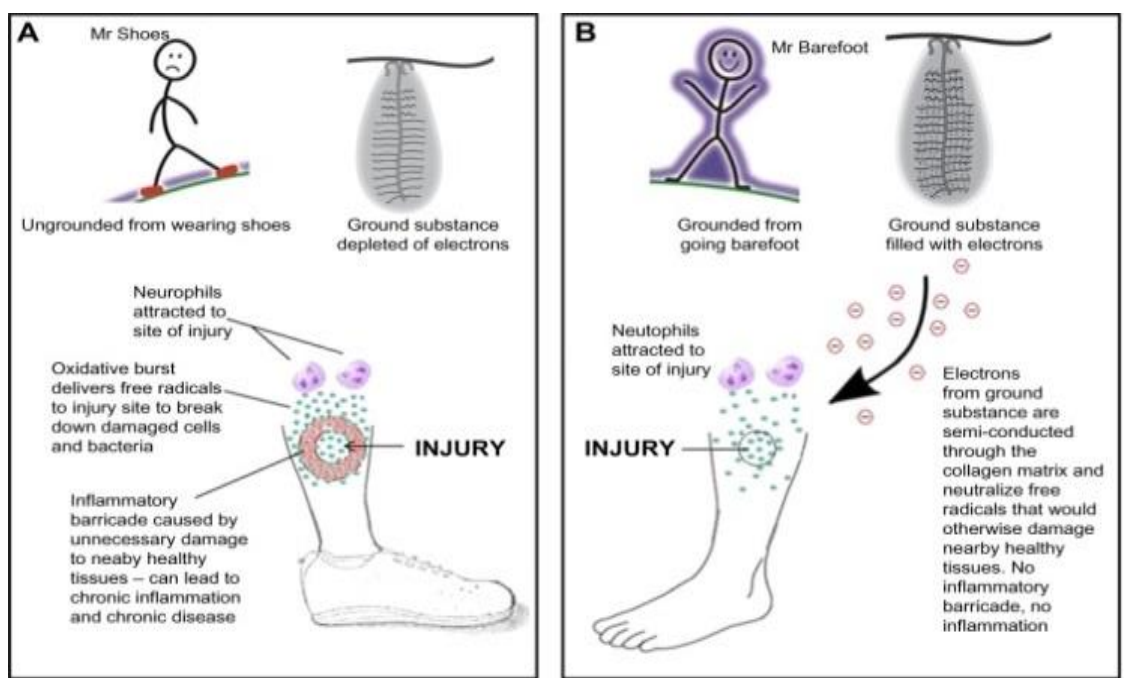

Fig. 6. Summary of the central hypothesis of Oschman et al. Notes: (A) After an injury, the ungrounded person (Mr. Shoes) will form an inflammatory barricade around the injury site. (B) After an injury, the grounded person (Mr. Barefoot) will not create an inflammatory barricade because reactive oxygen species could damage nearby healthy tissue (collateral damage) immediately neutralized by electrons semi-conducted electron-saturated ground substance via the collagen network.

In asthma, allergic rhinitis, pruritus, or other allergies, all of these disorders have an essential physio-pathological history and have been treated successfully with acupressure alone [23]-[24].

Both acupressure and grounding have dominated analgesic effects. Three studies examined the impact of grounding on delayed muscle soreness (DOMS). After unaccustomed or strenuous exercise, muscles felt pain, tenderness, and stiffness for several hours to days and concluded that this technique substantially decreased pain and inflammation [25]-[26].

Oschman described that earthing's immediate impact could not be explained by the slow movement of nerve impulses or chemical processes but rather by the inflow of antiinflammatory electrons, which can quickly transit through the conductive infrastructure to target inflammatory sites of the body [8,] [21].

Kirca et al. showed that acupressure had significant analgesic effects on postpartum perineal pain compared to the ice pack [25]. A systematic review of the treatment of various kinds of pain (dysmenorrhea, the pain of labour, low back pain, chronic headaches, and other traumatic pain) has demonstrated the efficacy of acupressure [27].

The improvement of blood circulation is another significant, documented benefit of grounding. In a study in 2013, the researchers concluded that grounding is one of the most simple but profound interventions to reduce cardiovascular risk and cardiovascular events."

It may be because the negative charge of the erythrocytes (the Zeta potential) increases and further create greater distance between the blood elements [28].

Two other studies have measured the increase in blood flow. The first study developed visual evidence showing that grounding enhances facial blood flow [29]. In further research, thermal imaging explains that grounding enhanced blood flow in the torso [30].

$\mathrm{Li}$ et al. assessed the effects of acupressure on the lower limb's blood flow for treating peripheral arterial occlusive diseases. They concluded that the lower limb blood flow in Stage II peripheral arterial occlusive diseases (PAOD) patients increased significantly by acupressure [31].
This technique has a significant impact on the autonomic nervous system (ANS). A study of premature infants has shown that grounding has resulted in immediate and substantial improvement in ANS measurements, which are important in controlling inflammatory responses and stress responses in those babies [32]. It may be because of the rapid transit movement of the electron that triggers the nerve vagus. However, acupuncture can effectively decrease gastric tachyarrhythmias and prevent paroxysmal supraventricular tachycardia; and vagus nerve stimulation [33].

\section{CONCLUSION}

Both acupressure and grounding are simple, non-invasive, and accessible techniques that, when combined, could have a significant and beneficial role in the regulation or treatment of inflammation, pain, circulation, and autonomic dysfunctions.

A device that could use both techniques simultaneously poses a tremendous opportunity to:

(a) increase the results of a typical acupressure session through dramatically increased, targeted, and controlled ion passage through and around the meridian point;

(b) enhancing the effects of grounding through allowing for a pinpointed ion gateway;

(c) might be helpful to in potentiating their biological and clinical benefits, while reducing costs at the same time.

This model also explains why, through repeated treatment, functional and organic, pathology can respond and resolve in many cases. Essentially, an acupuncture point is expressing when it becomes active (in other words, where disease, functional or organic, is present) is trying to resolve biophysically the electrical abnormality produced by the injury or illness in the affected tissue. The use of a biphasic current over the affected point facilitates this process, leading to the original pathology's resolution. 


\section{REFERENCES}

[1] Luo D, Wang X, He J. A comparison between acute pressure block of the sciatic nerve and acupressure: methodology, analgesia, and mechanism involved. Journal of pain research. 2013;6:589.

[2] Tournaire M, Theau-Yonneau A. Complementary and alternative approaches to pain relief during labor. Evidence-based complementary and alternative medicine. 2007;4(4):409-17.

[3] Narongpunt V, Datcu S, Ibos L, Adnet F, Fontas B, Candau Y, et al. Monitoring acupressure stimulation effects by infrared thermography. Quantitative InfraRed Thermography Journal. 2004;1(2):185-204.

[4] Cook A, Wilcox G. PRESSURING PAIN alternative therap for labor pain management. AWHONN lifelines. 1997;1(2):36-41.

[5] Langevin HM, Wayne PM. What is the point? The problem with acupuncture research that no one wants to talk about. The Journal of Alternative and Complementary Medicine. 2018;24(3):200-7.

[6] Mehta P, Dhapte V, Kadam S, Dhapte V. Contemporary acupressure therapy: Adroit cure for painless recovery of therapeutic ailments. Journal of traditional and complementary medicine. 2017;7(2):251-63.

[7] Lu A-P, Jia H-W, Xiao C, Lu Q-P. Theory of traditional Chinese medicine and therapeutic method of diseases. World journal of gastroenterology: WJG. 2004;10(13):1854.

[8] Oschman JL, Chevalier G, Brown R. The effects of grounding (earthing) on inflammation, the immune response, wound healing, and prevention and treatment of chronic inflammatory and autoimmune diseases. Journal of Inflammation Research. 2015;8:83.

[9] Park GY, Kwon DR, Moon YS. Low-intensity microcurrent therapy promotes the regeneration of atrophied calf muscles in immobilized rabbits. Journal of biomedical research. 2019;33(1):30.

[10] Picker R. Current trends: low-volt pulsed microamp stimulation, part I. Clin Manag Phys Ther. 1989;9(10-14):482.

[11] Picker RI. Low-volt pulsed microamp stimulation. Part II Clinical Management. 1989;9:28-33.

[12] Madill P. The Uses and Limitations of Acupuncture Point Measurement, German Electroacupuncture or Electroacupuncture According to Voll (EAV). American Journal of Acupuncture. 1984;12(1):33-42.

[13] Chen K-G. Electrical properties of meridians. IEEE Engineering in Medicine and Biology Magazine. 1996;15(3):58-63.

[14] Tan H, Tumilty S, Chapple C, Liu L, McDonough S, Yin H, et al. Understanding acupoint sensitization: a narrative review on phenomena, potential mechanism, and clinical application. EvidenceBased Complementary and Alternative Medicine. 2019;2019.

[15] Ulett GA, Han J, Han S. Traditional and evidence-based acupuncture. South Med J. 1998;91:1115-20.

[16] Omura Y. Electro-acupuncture: its electrophysiological basis and criteria for effectiveness and safety-part I. Acupuncture \& ElectroTherapeutics Research. 1975;1(1-2):157-81.

[17] Pethig R, Szent-Györgyi A. Bioelectrochemistry, the living state, and electronic conduction in proteins. Bioelectrochemistry: Springer; 1980. p. 227-52.

[18] Colthurst J, Giddings P. A retrospective case note review of the Fenzian electrostimulation system: a novel non-invasive, non-pharmacological treatment. The Pain Clinic. 2007;19(1):7-14.

[19] Sokal K, Sokal P. Earthing the human body influences physiologic processes. The Journal of Alternative and Complementary Medicine. 2011;17(4):301-8.

[20] Melzack R, Katz J. The gate control theory: Reaching for the brain. Pain: psychological perspectives. 2004:13-34.

[21] Oschman JL. Perspective: assume a spherical cow: the role of free or mobile electrons in bodywork, energetic and movement therapies. Journal of Bodywork and Movement Therapies. 2008;12(1):40-57.

[22] Oschman JL. Charge transfer in the living matrix. Journal of Bodywork and Movement Therapies. 2009;13(3):215-28.

[23] Liang Y, Lenon GB, Yang AWH. Self-administered acupressure for allergic rhinitis: study protocol for a randomized, single-blind, nonspecific controlled, parallel trial. Trials. 2019;20(1):1-8.

[24] Rehman IU, Chia DWB, Ahmed R, Khan NA, Rahman AU, Munib S, et al. A randomized controlled trial for effectiveness of zolpidem versus acupressure on sleep in hemodialysis patients having chronic kidney disease-associated pruritus. Medicine. 2018;97(31).

[25] Kirca AS, Gul DK. The effect of acupressure applied to points LV4 and LI4 on perceived acute postpartum perineal pain after vaginal birth with episiotomy: a randomized controlled study. Archives of gynecology and obstetrics. 2020;301(2):473-81.

[26] Müller E, Pröller P, Ferreira-Briza F, Aglas L, Stöggl T. Effectiveness of grounded sleeping on recovery after intensive eccentric muscle loading. Frontiers in physiology. 2019;10:35.
[27] Chen Y-W, Wang H-H. The effectiveness of acupressure on relieving pain: a systematic review. Pain Management Nursing. 2014;15(2):53950.

[28] Chevalier G, Sinatra ST, Oschman JL, Delany RM. Earthing (grounding) the human body reduces blood viscosity - a major factor in cardiovascular disease. The journal of alternative and complementary medicine. 2013;19(2):102-10.

[29] Chevalier G. Grounding the human body improves facial blood flow regulation: results of a randomized, placebo-controlled pilot study. Journal of Cosmetics, Dermatological Sciences, and Applications. 2014;4(05):293.

[30] Chevalier G, Melvin G, Barsotti T. One-hour contact with the Earth's surface (grounding) improves inflammation and blood flow-A randomized, double-blind, pilot study. Health. 2015;7(08):1022.

[31] Li X, Hirokawa M, Inoue Y, Sugano N, Qian S, Iwai T. Effects of acupressure on lower limb blood flow for the treatment of peripheral arterial occlusive diseases. Surgery Today. 2007;37(2):103-8.

[32] Passi R, Doheny KK, Gordin Y, Hinssen H, Palmer C. Electrical grounding improves vagal tone in preterm infants. Neonatology. 2017;112(2):187-92.

[33] Zhao F, Cui S, Zheng M. Termination of paroxysmal supraventricular tachycardia with P6 acupressure. Journal of anesthesia. 2014;28(4):644-.

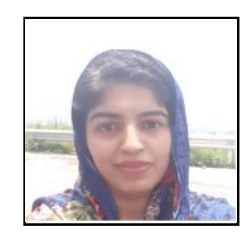

\section{Madiha Khalid}

Place of birth: Lahore, India.

Date of Birth: 11/9/1985.

M. Phil clinical Pharmacy.

Humdard University, Lahore.

She is working as a lecturer in the University of

Lahore for about 6 years in the Pharmacy Department.

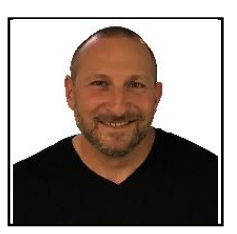

\section{Jeremy Madvin}

Place of Birth: Los Angeles, United States.

Bate of Birth: 11/22/1967.

MBA International Business. 